Supplement of Hydrol. Earth Syst. Sci., 21, 2127-2142, 2017

http://www.hydrol-earth-syst-sci.net/21/2127/2017/

doi:10.5194/hess-21-2127-2017-supplement

(C) Author(s) 2017. CC Attribution 3.0 License.

(c) (1)

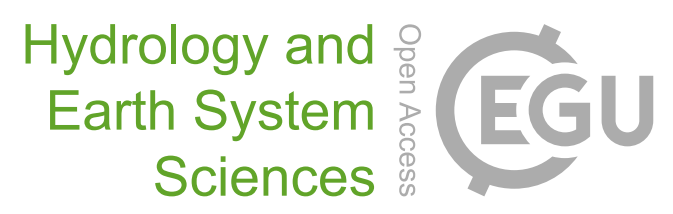

Supplement of

\title{
Temporal and spatial changes of rainfall and streamflow in the Upper Tekezē-Atbara river basin, Ethiopia
}

Tesfay G. Gebremicael et al.

Correspondence to: Tesfay G. Gebremicael (t.gebremicael@unesco-ihe.org, dutg2006@gmail.com)

The copyright of individual parts of the supplement might differ from the CC-BY 3.0 licence. 
Table S1. Summary results of MK, Z statistics on monthly rainfall trends. Negative/positive Z value indicates a decreasing/increasing trend and in bold a statistical significant trend at $5 \%$ confidence level $(Z= \pm 1.96)$.

\begin{tabular}{|c|c|c|c|c|c|c|c|c|c|c|c|c|c|}
\hline Station & Statistics & Jan & Feb & Mar & Apr & May & Jun & Jul & Aug & Sep & Oct & Nov & Dec \\
\hline \multirow[t]{2}{*}{ Makele (AP) } & $\mathrm{Z}$ & -1.04 & -0.57 & 0.54 & -1.56 & 0.44 & 1.07 & -0.38 & -1.45 & -1.40 & 2.10 & 1.67 & 5.45 \\
\hline & $\mathrm{P}$ & 0.30 & 0.56 & 0.60 & 0.13 & 0.66 & 0.30 & 0.57 & 0.39 & 0.16 & 0.04 & 0.1 & 0.00 \\
\hline \multirow[t]{2}{*}{ Adigrat } & $\mathrm{Z}$ & 0.95 & -0.66 & 0.32 & -0.23 & 1.16 & 0.48 & -1.18 & -1.07 & -1.41 & 0.56 & -0.17 & 1.99 \\
\hline & $\mathrm{P}$ & 0.34 & 0.51 & 0.79 & 0.82 & 0.25 & 0.65 & 0.24 & 0.33 & 0.39 & 0.58 & -0.09 & 0.04 \\
\hline \multirow[t]{2}{*}{ Mychew } & $\mathrm{Z}$ & 1.90 & -2.86 & -1.14 & -0.56 & 1.13 & 0.94 & 0.32 & 0.35 & 0.26 & 0.68 & 2.24 & -0.28 \\
\hline & $\mathrm{P}$ & 0.06 & 0.02 & 0.26 & 0.58 & 0.26 & 0.35 & 0.75 & 0.73 & 0.80 & 0.50 & 0.026 & 0.78 \\
\hline \multirow[t]{2}{*}{ Shire } & $\mathrm{Z}$ & 0.82 & -0.64 & 1.32 & -0.38 & 1.32 & 2.64 & -0.65 & 0.28 & 2.29 & 0.28 & 0.52 & 0.18 \\
\hline & $\mathrm{P}$ & 0.41 & 0.53 & 0.19 & 0.68 & 0.21 & 0.02 & 0.63 & 0.74 & 0.02 & 0.77 & 0.61 & 0.86 \\
\hline \multirow[t]{2}{*}{ D/tabour } & $\mathrm{Z}$ & 2.44 & 2.11 & 1.83 & 0.72 & 0.69 & -0.04 & -1.90 & -1.93 & -0.55 & 0.14 & 2.17 & 3.59 \\
\hline & $\mathrm{P}$ & 0.05 & 0.04 & 0.06 & 0.47 & 0.48 & 0.95 & 0.07 & 0.07 & 0.54 & 0.21 & 0.03 & 0.00 \\
\hline \multirow[t]{2}{*}{ Mykinetal } & $\mathrm{Z}$ & -2.95 & -2.40 & -0.96 & -1.90 & -0.50 & 0.49 & -1.79 & 1.21 & 2.60 & -0.72 & -1.19 & -2.36 \\
\hline & $\mathrm{P}$ & 0.003 & 0.02 & 0.34 & 0.06 & 0.62 & 0.55 & 0.08 & 0.20 & 0.01 & 0.50 & 0.24 & 0.04 \\
\hline \multirow[t]{2}{*}{ Gonder } & $\mathrm{Z}$ & -1.49 & 0.15 & -1.41 & 0.59 & 0.25 & 1.01 & 0.28 & 0.13 & 0.52 & 0.17 & -1.66 & -1.79 \\
\hline & $\mathrm{P}$ & 0.14 & 0.88 & 0.16 & 0.54 & 0.70 & 0.41 & 0.78 & 0.87 & 0.66 & 0.87 & 0.10 & 0.08 \\
\hline \multirow[t]{2}{*}{ Adigudem } & $\mathrm{Z}$ & 4.98 & -2.56 & 0.22 & 0.98 & -0.47 & -0.68 & 0.36 & 0.21 & 0.24 & -0.23 & 3.95 & 5.26 \\
\hline & $\mathrm{P}$ & 0.001 & 0.01 & 0.83 & 0.09 & 0.65 & 0.16 & 0.62 & 0.16 & 0.81 & 0.82 & 0.001 & 0.001 \\
\hline \multirow[t]{2}{*}{$\mathrm{H} / \mathrm{selam}$} & $\mathrm{Z}$ & 0.00 & -1.13 & -1.65 & -2.99 & 0.48 & -0.27 & -0.62 & 0.19 & 0.12 & -0.51 & -0.58 & 1.42 \\
\hline & $\mathrm{P}$ & 0.98 & 0.001 & 0.23 & 0.68 & 0.65 & 0.75 & 0.11 & 0.80 & 0.92 & 0.56 & 0.16 & 0.03 \\
\hline \multirow[t]{2}{*}{ Hawzen } & $\mathrm{Z}$ & -0.48 & -1.60 & -0.51 & -0.55 & -0.42 & 0.08 & -0.08 & 0.37 & 0.07 & -0.91 & 0.00 & -1.13 \\
\hline & $\mathrm{P}$ & 0.11 & 0.06 & 0.55 & 0.58 & 0.40 & 0.93 & 0.92 & 0.40 & 0.92 & 0.36 & 1.0 & 0.26 \\
\hline \multirow[t]{2}{*}{ Wukro } & $\mathrm{Z}$ & -6.15 & -3.79 & -1.48 & -0.90 & -1.95 & 1.11 & 0.10 & 1.10 & -1.09 & -0.88 & -1.30 & -2.60 \\
\hline & $\mathrm{P}$ & 0.001 & 0.001 & 0.14 & 0.35 & 0.06 & 0.22 & 0.75 & 0.22 & 0.38 & 0.38 & 0.20 & 0.01 \\
\hline \multirow[t]{2}{*}{ Abiadi } & $\mathrm{Z}$ & -1.56 & -1.90 & -2.27 & -2.27 & -1.85 & 1.78 & 0.72 & 0.32 & 1.73 & -0.58 & -0.40 & 2.98 \\
\hline & $\mathrm{P}$ & 0.12 & 0.06 & 0.02 & 0.02 & 0.06 & 0.06 & 0.10 & 0.14 & 0.06 & 0.56 & 0.71 & 0.00 \\
\hline \multirow[t]{2}{*}{ Demgolat } & $\mathrm{Z}$ & -1.47 & -3.44 & 0.05 & -0.08 & 1.09 & 0.03 & 0.13 & 1.82 & 0.01 & -2.12 & -0.48 & -2.42 \\
\hline & $\mathrm{P}$ & 0.14 & 0.001 & 0.95 & 0.86 & 0.31 & 0.97 & 0.83 & 0.06 & 0.99 & $\mathbf{0 . 0 3}$ & 0.63 & 0.02 \\
\hline \multirow[t]{2}{*}{ E/hamus } & $\mathrm{Z}$ & -1.63 & -1.10 & -2.45 & -1.62 & -1.02 & -0.95 & 0.21 & -0.64 & -0.59 & -0.86 & 0.33 & -1.58 \\
\hline & $\mathrm{P}$ & 0.10 & 0.04 & 0.02 & 0.08 & 0.31 & 0.39 & 0.85 & 0.53 & 0.56 & 0.39 & 0.74 & 0.08 \\
\hline \multirow[t]{2}{*}{ Adwa } & $\mathrm{Z}$ & 0.25 & -0.76 & 0.24 & 0.33 & 1.01 & 0.96 & 0.81 & 2.19 & 1.32 & 0.35 & 1.13 & 0.48 \\
\hline & $\mathrm{P}$ & 0.68 & 0.45 & 0.81 & 0.74 & 0.30 & 0.29 & 0.23 & 0.01 & 0.12 & 0.73 & 0.27 & 0.09 \\
\hline \multirow[t]{2}{*}{ Axum } & $\mathrm{Z}$ & -1.64 & -3.52 & -1.09 & 0.20 & 0.12 & 0.61 & -0.72 & 0.91 & 2.25 & -1.26 & -0.02 & 0.53 \\
\hline & $\mathrm{P}$ & 0.10 & 0.00 & 0.32 & 0.81 & 0.83 & 0.43 & 0.48 & 0.26 & 0.03 & 0.21 & 0.98 & 0.60 \\
\hline \multirow[t]{2}{*}{ Debark } & $\mathrm{Z}$ & 1.34 & -2.94 & 1.70 & 0.31 & 0.82 & 1.20 & 1.92 & 0.51 & -0.43 & -0.97 & -1.11 & 0.17 \\
\hline & $\mathrm{P}$ & 0.18 & -0.001 & 0.12 & 0.61 & 0.44 & 0.09 & 0.06 & 0.36 & 0.51 & 0.33 & 0.29 & 0.87 \\
\hline \multirow[t]{2}{*}{ Lalibela } & $\mathrm{Z}$ & 1.10 & $\begin{array}{l}1.74 \\
\end{array}$ & 0.85 & -0.22 & 1.28 & 1.32 & 1.17 & 2.59 & 4.41 & 0.71 & 0.06 & 0.67 \\
\hline & $\mathrm{P}$ & 0.27 & 0.05 & 0.40 & 0.83 & 0.23 & 0.20 & 0.11 & 0.01 & 0.001 & 0.48 & 0.95 & 0.01 \\
\hline \multirow[t]{2}{*}{ Samre } & $\mathrm{Z}$ & -6.68 & -1.22 & -0.54 & -0.14 & $\begin{array}{l}-0.68 \\
\end{array}$ & -2.21 & -0.08 & -0.04 & 0.89 & -0.29 & -1.04 & -2.17 \\
\hline & $P$ & 0.001 & $\mathbf{0 . 0 3}$ & 0.59 & 0.90 & 0.50 & 0.03 & 0.91 & 0.95 & 0.37 & 0.77 & 0.30 & 0.03 \\
\hline
\end{tabular}


5 Table S2. Summary results of Pettitt test on seasonal rainfall. Negative/positive K value indicates a decreasing/increasing trend and in bold a statistical significant trend at $5 \%$ confidence level.

\begin{tabular}{|c|c|c|c|c|c|}
\hline \multirow[t]{2}{*}{ Station name } & \multirow{2}{*}{$\begin{array}{l}\text { Statistical } \\
\text { indices }\end{array}$} & \multicolumn{4}{|c|}{ Periods/season } \\
\hline & & annual & Rainy & Dry season & Short rainy \\
\hline \multirow[t]{2}{*}{ Mekelle (AP) } & $\mathrm{K}$ & 122 & 60 & 129 & 200 \\
\hline & $P$ & 0.55 & 1.0 & 0.52 & 0.10 \\
\hline \multirow[t]{2}{*}{ Mychew } & $\mathrm{K}$ & 230 & 212. & 242 & 174 \\
\hline & $\mathrm{P}$ & 0.20 & 0.3 & 0.18 & 0.549 \\
\hline \multirow[t]{2}{*}{ Axum } & $\mathrm{K}$ & 242 & 168 & 148 & 100 \\
\hline & $\mathrm{P}$ & 0.04 & 0.30 & 0.44 & 0.85 \\
\hline \multirow[t]{2}{*}{ Gonder } & $\mathrm{K}$ & 131 & 126 & 146 & 122 \\
\hline & $P$ & 0.51 & 0.55 & 0.38 & 0.60 \\
\hline \multirow[t]{2}{*}{ Adwa } & $\mathrm{K}$ & 360 & 301 & 177. & 135 \\
\hline & $\mathrm{P}$ & 0.06 & 0.01 & 0.21 & 0.52 \\
\hline \multirow[t]{2}{*}{ Mykinetal } & $\mathrm{K}$ & 122 & 109 & 184 & 184. \\
\hline & $\mathrm{P}$ & 0.56 & 0.68 & 0.13 & 0.13 \\
\hline \multirow[t]{2}{*}{ Shire } & $\mathrm{K}$ & -242 & 178 & 184 & 152 \\
\hline & $\mathrm{P}$ & 0.03 & 0.12 & 0.11 & 0.25 \\
\hline \multirow[t]{2}{*}{ Adigrat } & $\mathrm{K}$ & 167 & 165 & 91 & 93 \\
\hline & $P$ & 0.20 & 0.21 & 0.85 & 0.83 \\
\hline \multirow[t]{2}{*}{ Adigudem } & $\mathrm{K}$ & 122 & 60 & 129 & 200 \\
\hline & $\mathrm{P}$ & 0.55 & 1.0 & 0.52 & 0.10 \\
\hline \multirow[t]{2}{*}{ Edagahamus } & $\mathrm{K}$ & 78 & 112 & 218 & 121 \\
\hline & $\mathrm{P}$ & 0.07 & 0.52 & 0.03 & 0.11 \\
\hline \multirow[t]{2}{*}{ Hawzen } & $\mathrm{K}$ & 96 & 184 & 218 & 201 \\
\hline & $\mathrm{P}$ & 0.83 & 0.16 & 0.06 & 0.10 \\
\hline \multirow[t]{2}{*}{ Hagereselam } & $\mathrm{K}$ & 122 & 118 & 112 & 105 \\
\hline & $\mathrm{P}$ & 0.50 & 0.08 & 0.61 & -0.70 \\
\hline \multirow[t]{2}{*}{ AbiAdi } & $\mathrm{K}$ & 150 & 176 & 112 & -176 \\
\hline & $\mathrm{P}$ & 0.06 & 0.08 & 0.09 & 0.07 \\
\hline \multirow[t]{2}{*}{ Debretabour } & $\mathrm{K}$ & 137 & 188 & 176 & 97 \\
\hline & $P$ & 0.34 & 0.05 & 0.06 & 0.65 \\
\hline \multirow[t]{2}{*}{ Dengolat } & $\mathrm{K}$ & 162 & 162 & 140 & 64 \\
\hline & $\mathrm{P}$ & 0.09 & 0.10 & 0.20 & 0.94 \\
\hline \multirow[t]{2}{*}{ Lalibela } & $\mathrm{K}$ & 72 & 64 & 108 & 127 \\
\hline & $\mathrm{P}$ & 0.28 & 0.41 & 0.33 & 0.13 \\
\hline \multirow[t]{2}{*}{ Wukro } & $\mathrm{K}$ & 100 & 158 & 230 & 186 \\
\hline & $\mathrm{P}$ & 0.42 & 0.05 & 0.09 & 0.11 \\
\hline \multirow[t]{2}{*}{ Kulmesk } & $\mathrm{K}$ & 16 & 14 & 20 & 32 \\
\hline & $\mathrm{P}$ & 0.65 & 0.77 & 0.41 & 0.05 \\
\hline \multirow[t]{2}{*}{ Debark } & $\mathrm{K}$ & 54 & 82 & 71 & 68 \\
\hline & $\mathrm{P}$ & 0.04 & 0.06 & 0.15 & 0.17 \\
\hline \multirow[t]{2}{*}{ Samre } & $\mathrm{K}$ & 184 & 118 & 85 & 67 \\
\hline & $\mathrm{P}$ & 0.16 & 0.64 & 0.07 & 0.05 \\
\hline
\end{tabular}




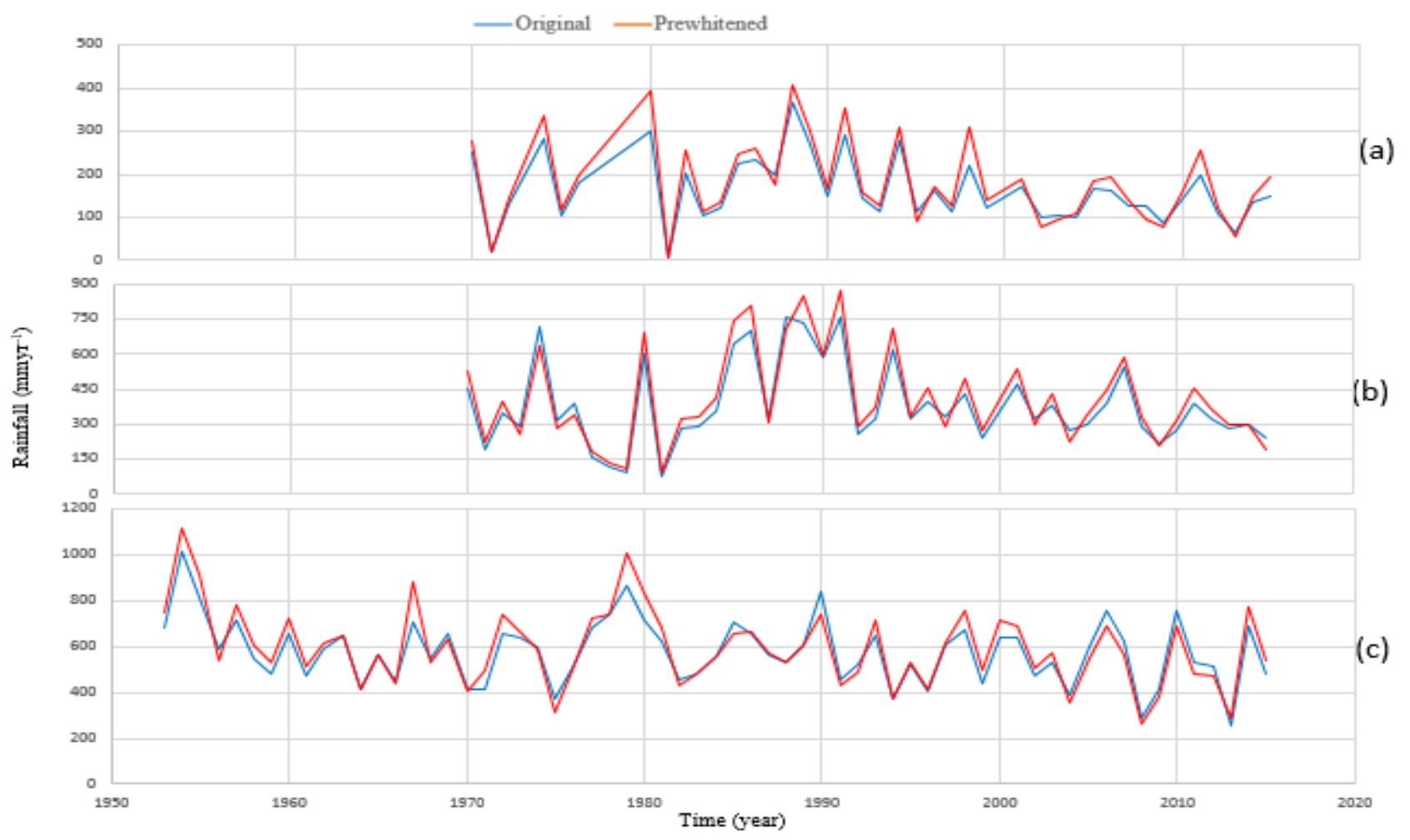

Figure. S1: Comparison of rainfall data before and after removal of auto-correlation: (a) monthly rainfall in Adigrat for August, (b) main rainy season in Adigrat, (c) annual rainfall in Mekelle (AP) 


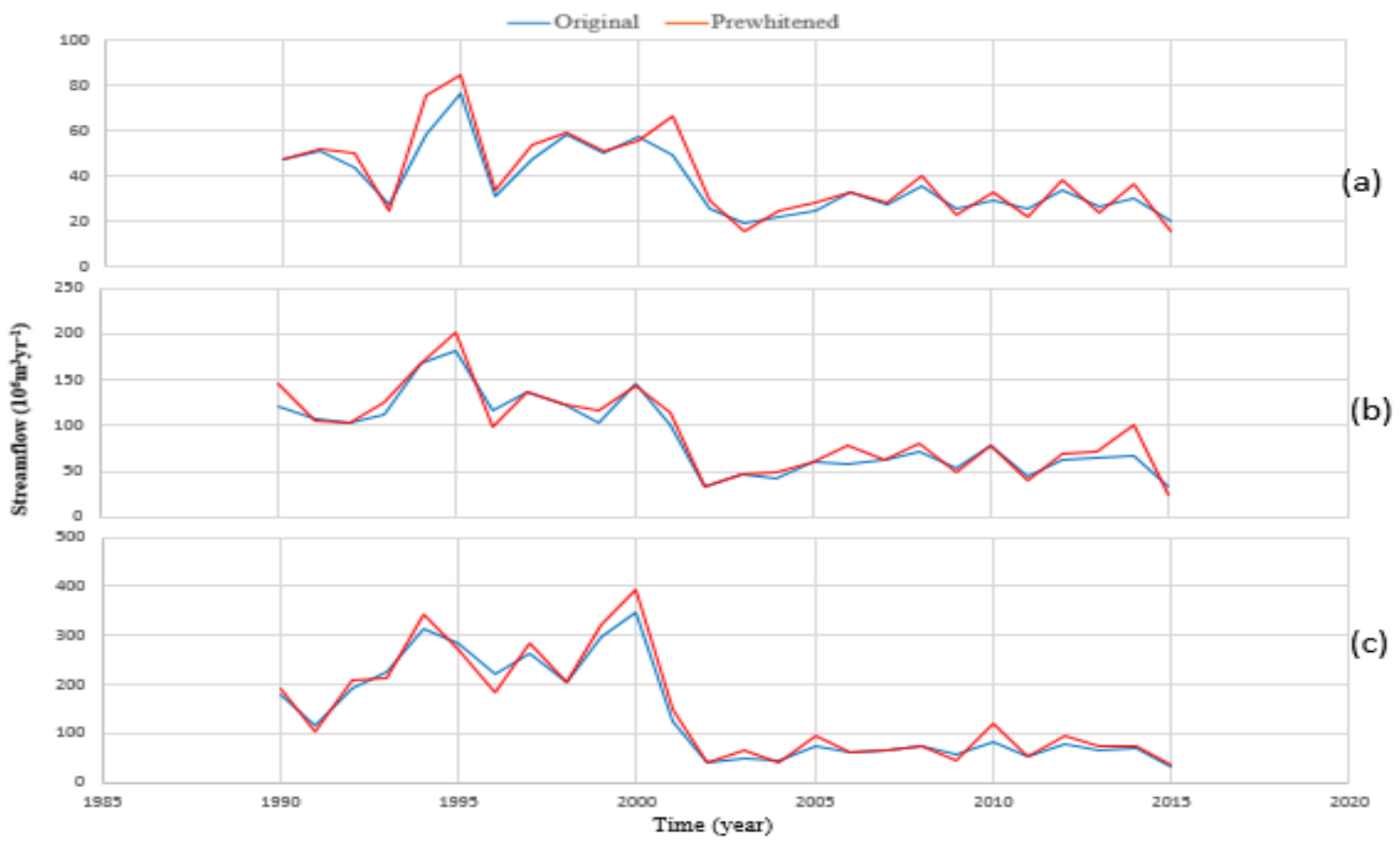

Figure. S2: Comparison of streamflow data before and after removal of auto-correlation at Geba 1 station: (a) monthly for August, (b) main rainy season, (c) annual 

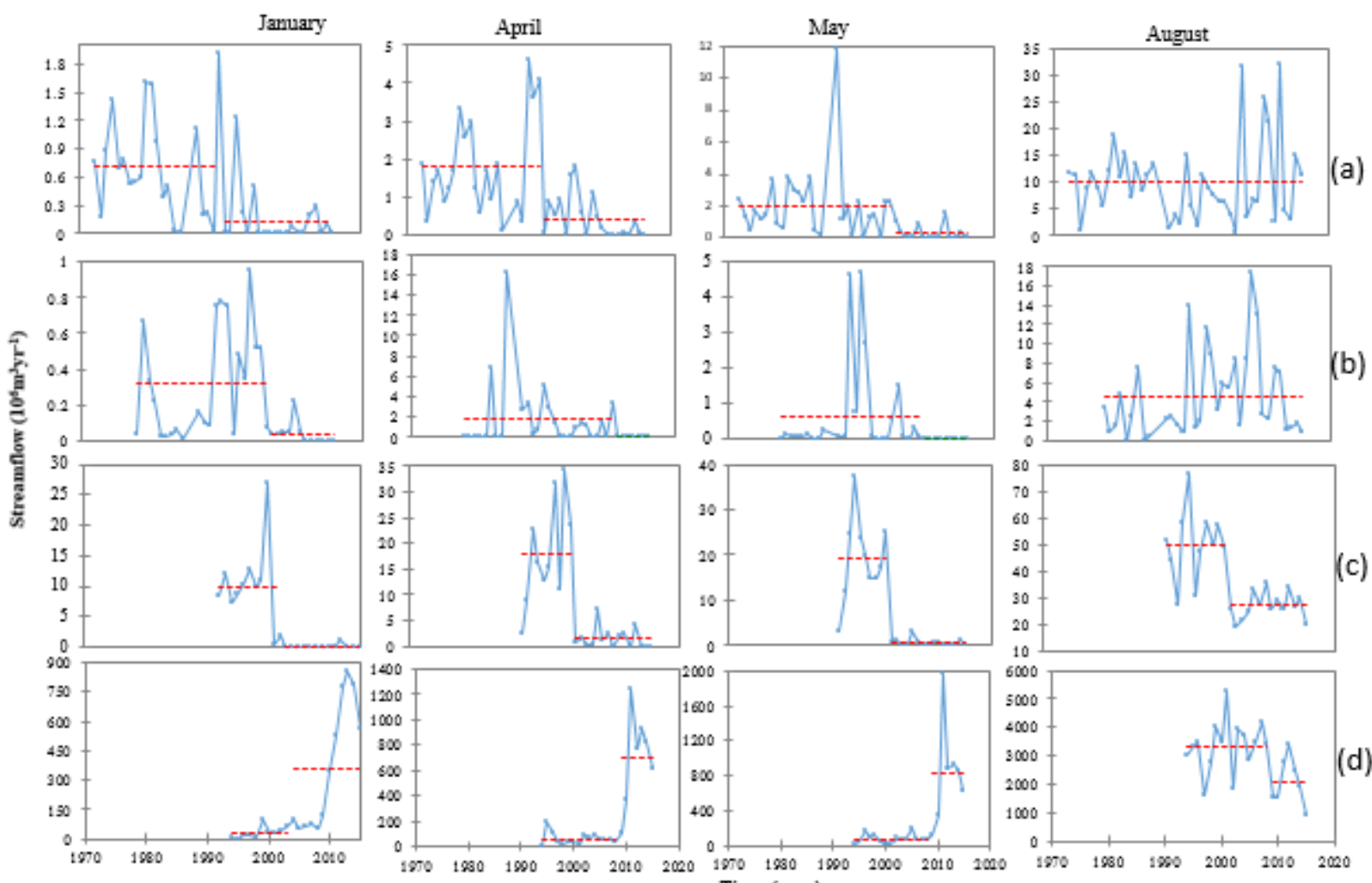

5

Figure S3. Pettitt homogeneity test on monthly streamflow for selected stations (a) Siluh, (b) Illala, (c) Geba 1, (d) Emabamadre. 